\title{
Variability in the anterior extralaryngeal branch of the recurrent laryngeal nerve: clinical implications
}

\author{
K. Hessel, E. Wessel, A. Olinger, B.W. Wright \\ Anatomy Fellow, Department of Anatomy, Kansas City University of Medicine and Biosciences, Kansas City, MO, United States \\ [Received: 18 June 2014; Accepted: 21 July 2014]
}

Background: This study aimed to identify the anterior and posterior extralaryngeal branches (AELB, PELB) of the recurrent laryngeal nerve (RLN), measure these branches when present, and determine relationships between gender, sidedness and neck length.

Materials and methods: Dissection was completed to level of the thyroid on 45 cadavers. The course of the RLN was then traced superiorly from its entry into the neck. Careful reflection of the thyroid and dissection of the lateral thyroid ligament permitted visualisation of the full course of the nerve. If extralaryngeal branching (ELB) was present, measurements were taken from the point of bifurcation of the RLN to the point of laryngeal entry through the cricothyroid membrane. Neck measurements, from the spinous process of $C 7$ to the superior nuchal line, were taken. Gender of the specimen was noted. Data was analysed in SPSS. Results: Extralaryngeal branching was found in $77.78 \%$ of our sample, $77.14 \%$ on the left and $54.29 \%$ on the right. A significant difference was found between $A E L B$ length on the left and right, indicating that the left branch will be longer than the right when present. A significant difference in neck length between those with and without ELB was also found, indicating that people with longer necks more often display ELB. Neither neck length and AELB length, nor gender and $A E L B$ length were strongly correlated in this sample.

Conclusions: Extralaryngeal branching can occur in all populations, but there are definite trends in its incidence and length. Surgeons should be aware of these trends before operating on patients. (Folia Morphol 2017; 75, 1: 44-50)

Key words: anterior extralaryngeal branch, posterior extralaryngeal branch, recurrent laryngeal nerve

\section{INTRODUCTION}

The recurrent laryngeal nerve (RLN) is a branch of the vagus nerve. The course of the right and left RLN differ within the neck. The left RLN tends to course within the tracheoesophageal groove in the neck, while the right RLN is more commonly identified based on its relationship to the inferior thyroid artery, with the nerve anterior to or among the branches of the artery [18]. While the location of the RLN is relatively well-known, the branching pattern of the RLN as it courses through the neck is not as well studied. This is pertinent information for any surgeon

Address for correspondence: A. Olinger, PhD, Assistant Professor, Department of Anatomy, Kansas City University of Medicine and Biosciences, Kansas City, MO 64106, United States, tel: 816-654-7535, e-mail: Aolinger@kcumb.edu 
performing a thyroidectomy or parathyroidectomy, as the RLN courses posteromedial to the thyroid gland and posterolateral to the lateral thyroid ligament $[2,9]$, which is dissected as part of the thyroidectomy. Surgeons, therefore, must be aware of the potential branching patterns of the RLN. Of particular interest are the extralaryngeal branches: the anterior (AELB) and posterior (PELB) extralaryngeal nerves. While the anatomy of the RLN has been known for centuries, the AELB and PELB were described for the first time in 1957 by Gregg [7]. An extralaryngeal branch (ELB) is defined as any branch that bifurcates from the RLN and pierces the cricothyroid membrane, just inferior to the cricopharyngeus muscle, to innervate the larynx (Fig. 1). In this study we assessed the incidence of ELB in our sample, then determined the influence of neck length, gender and sidedness on its incidence and on its length.

The significance of the AELB and PELB has been debated in the literature for many years. Previous studies have claimed that the anterior and posterior branches carry adductor and abductor fibres, respectively $[7,15]$. Other studies have shown that branches from the AELB run to both adductor and abductor muscles [20, 22]. Still more studies have argued that the branches actually contain separate sensory and motor divisions [10, 18]. In 2009, Serpell et al. [18], tested this theory using nerve stimulation during thyroidectomy to measure the contraction of the laryngeal constrictors when either the AELB or PELB was stimulated. They found that when the AELB was stimulated, laryngeal constriction occurred. This led to the belief that the motor fibres to the laryngeal constrictors were being carried entirely by the AELB of the RLN when this branch was present $[10,18]$. Though damage to the RLN during thyroidectomy is rare, there is greater risk of damage when ELB is present but unidentified. If the AELB is severed without knowledge, the result is the same as if the RLN was damaged; deinnervation of the laryngeal constrictors. Paralysis of the laryngeal musculature results in detrimental side effects for the patient, including hoarseness and (if paralysis is bilateral) collapse of the vocal folds, aphonia and potential suffocation.

The AELB and PELB have been described in several studies using surgical patients undergoing thyroidectomy $[2-5,9-12,16,18]$, and in cadaveric samples $[13,15,17,19,21]$. Though there are some drawbacks to using cadaveric specimens, such as the inability to use a neurostimulator, there are several

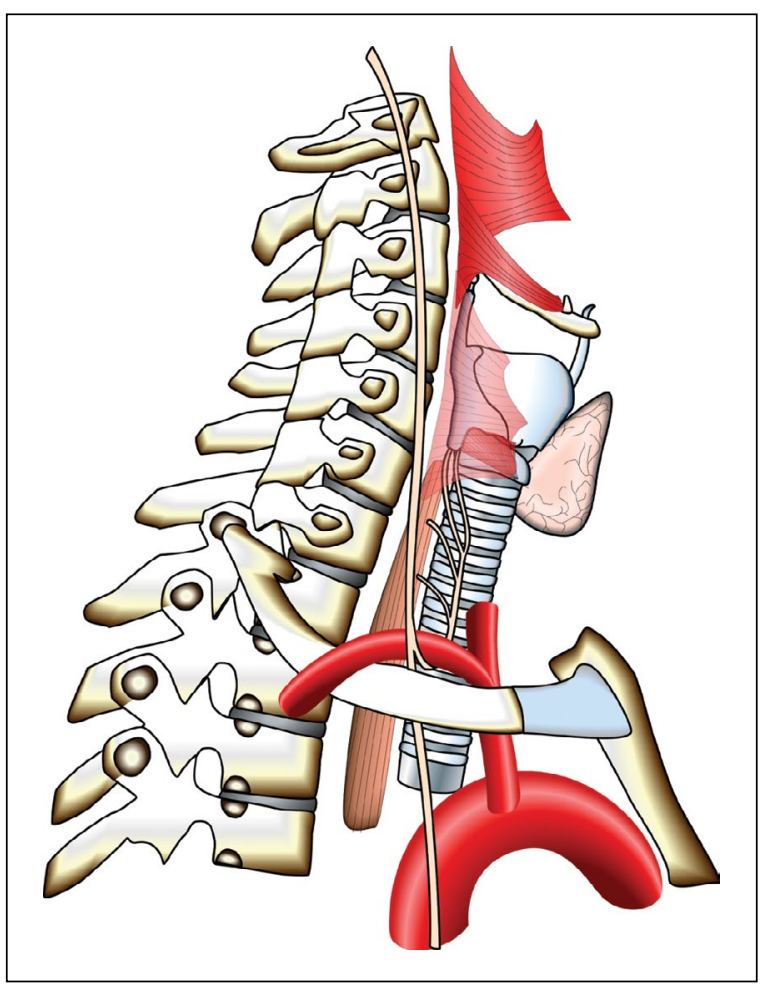

Figure 1. Schematic of the anterior and posterior extralaryngeal branches bifurcating from the recurrent laryngeal nerve and piercing the cricothyroid membrane beneath the cricopharyngeus muscle.

benefits to using cadaveric specimens. One of these benefits is the ability to completely dissect a nerve without risk of damage to the surrounding structures. In addition, our cadaveric sample includes a relatively equal male to female ratio for comparison between genders. This is not the case in most of the surgical samples previously studied, where the female to male ratio was often greater than $3: 1$, due to the higher incidence of thyroid disease in women $[4,10-12$, $16,18]$. The relative equality between genders in our sample allowed us to accurately compare incidence and length of AELB between genders. We also chose to exclude those specimens with a previous history of thyroid disease from our study. The exclusion of those with thyroid disease is of value because patients with goitre or large cancerous nodules may exhibit stretching of the RLN, resulting in an overestimation of the average AELB length.

The distance from the bifurcation of the AELB and PELB to the cricothyroid membrane is of particular surgical relevance since this distance may influence the approach during thyroidectomy. The ELB has previously been reported to occur approximately 


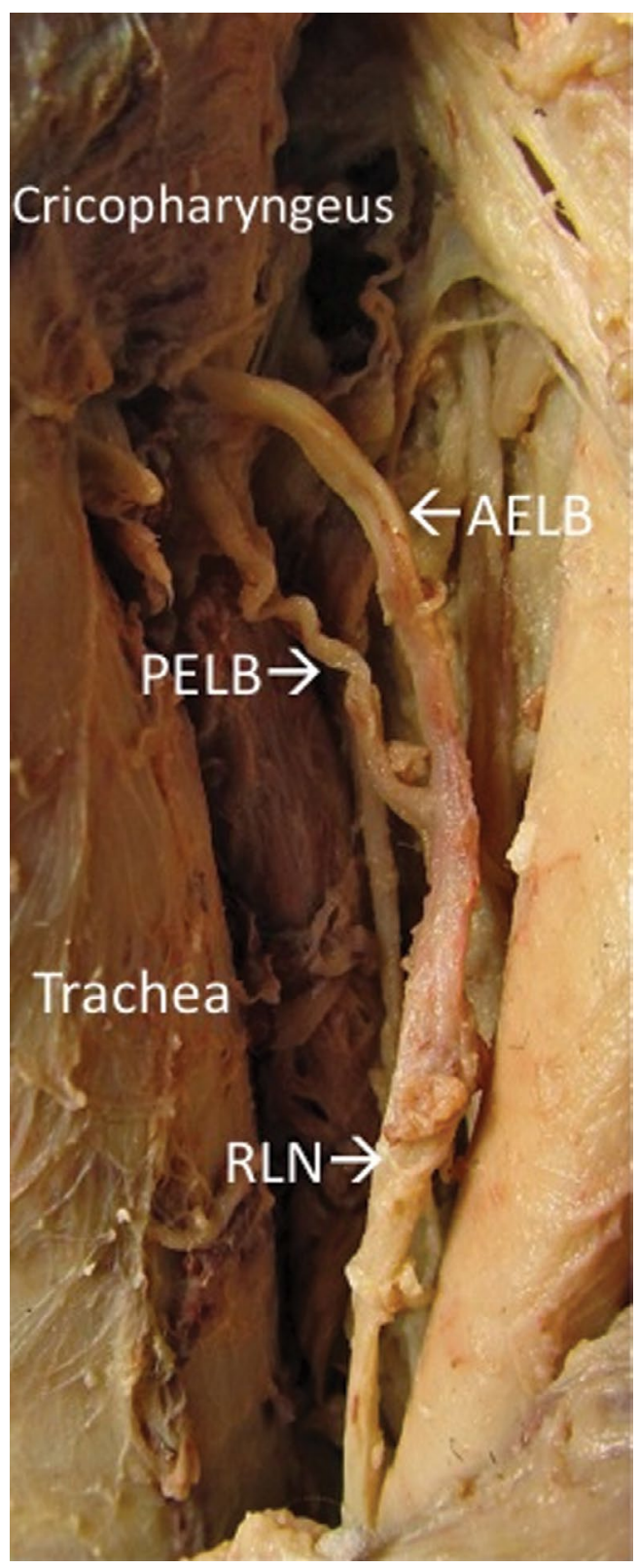

Figure 2. Left recurrent laryngeal nerve (RLN) bifurcating into anterior and posterior extralaryngeal branches (AELB, PELB), prior to piercing the cricothyroid membrane just inferior to the cricopharyngeus muscle.

$2 \mathrm{~cm}$ from the entry point of the nerve into the larynx beneath the cricopharyngeus muscle $[3,11,16-19]$. We were interested in noting whether the general asymmetry between the right and left RLN would extend to the ELB as well. There was also the possibility that gender would significantly impact the location of bifurcation because changes in the endocrine environment during puberty induce the anteroposterior dimension of the thyroid cartilage to undergo

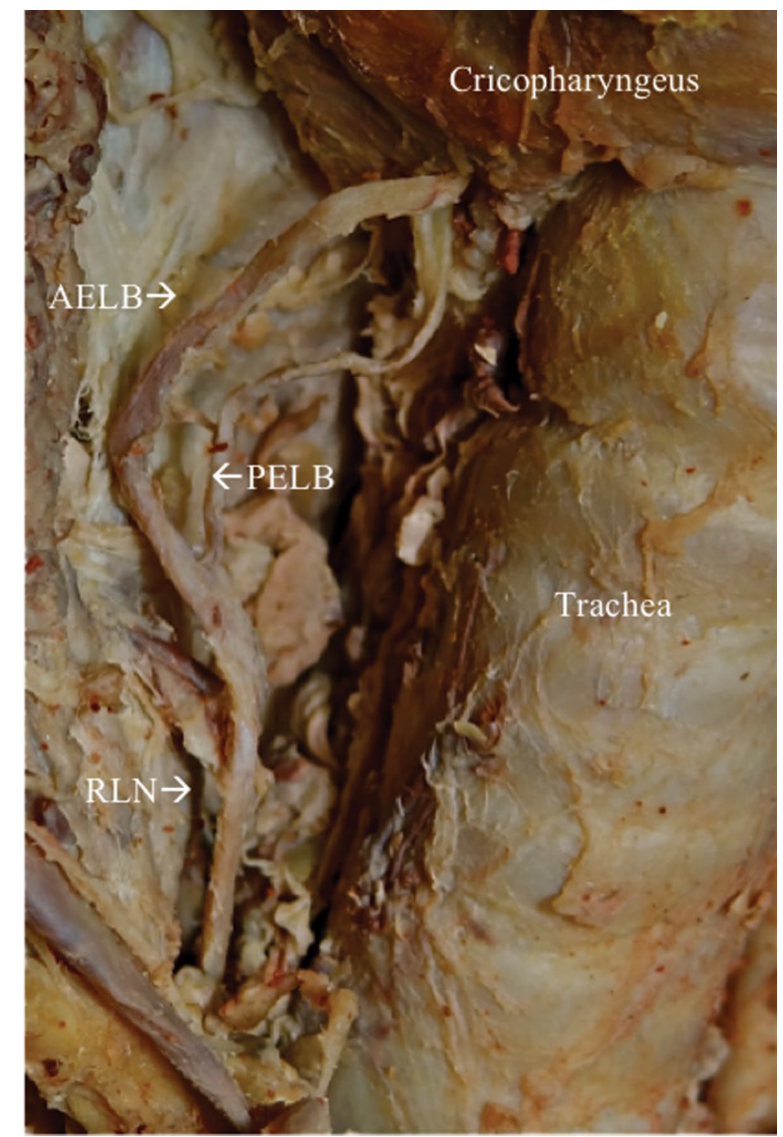

Figure 3. Right recurrent laryngeal nerve (RLN) bifurcating into anterior and posterior extralaryngeal branches (AELB, PELB) and piercing the cricothyroid membrane inferior to the cricopharyngeus muscle.

3 times more growth in males than in females [8]. Overall, increases in length, height, and width of the cartilaginous skeleton of the larynx is greater in men than women $[6,8,14]$, indicating that were the AELB present, it would be longer in males as a result of laryngeal growth during puberty.

It should be noted that previous studies did not consider any nerves that branched within $5 \mathrm{~mm}$ of the cricothyroid membrane to be ELB as most RLNs will begin to fan out in the terminal $5 \mathrm{~mm}$ of the nerve $[3,9,11,12,18]$. Therefore, our study only considered those branches exiting the nerve prior to the $5 \mathrm{~mm}$ mark as extralaryngeal.

We were additionally interested in determining whether an individual's neck length is itself a contributing factor in the incidence and branch point of the AELB. Identifying a relationship between any of these variables and the presence and position of the AELB would be of great benefit to surgeons, providing predictors for ELB in their surgical patients. 


\section{MATERIALS AND METHODS}

Ninety RLNs from 44 human cadavers were obtained. Cadavers were embalmed. Data was collected from 20 female and 25 male cadavers. Those with a history of thyroid disease, thyroidectomy, or any evidence of palpable thyroid nodules were excluded from this study.

All specimens were dissected and measured by the same individual in order to eliminate interobserver error. Dissection consisted of skinning the neck to reveal the platysma muscle, which was then reflected superolaterally, as was the sternocleidomastoid muscle. The carotid sheath was drawn laterally, and the infrahyoid muscles were reflected superiorly. The RLN was then identified as it entered the neck and coursed superiorly. Once the RLN was located, the isthmus of the thyroid was cut at the midline and the thyroid was carefully reflected laterally, with particular care taken during the dissection of the lateral thyroid ligament. The RLN was followed until its entrance into the larynx through the cricothyroid membrane, just inferior to the cricopharyngeus muscle. Fine dissection was used to determine if multiple branches were present. If an ELB was found, measurement of the AELB from the point of bifurcation to the point of entry into the larynx was taken, using digital Mitutoyo callipers. Two measurements of the nerve were taken and the average calculated. We found that the difference between the two measurements for all individuals was negligible $(<0.05 \%)$, so the average of the two was used for analysis. Gender was recorded for all specimens. The cadaver was then put into the prone position, with a $4 \times 4$ block placed under the thorax to allow the neck flexion necessary to compensate for any head extension due to post-embalming muscle rigidity and cadaver positioning. To ensure that all of the necks were in the same state of flexion, pins were inserted into the lateral aspect of the posterior tubercle of the transverse process of $\mathrm{C} 1$ and $\mathrm{C}$. The pins were then levelled using a spirit level and held in place while the neck length was measured. Total neck length was obtained by measuring from the inferior edge of the spinous process of $\mathrm{C} 7$ to the superior nuchal line on the occipital bone. Two measurements were again taken and averaged to increase reliability. Twenty-seven of 45 cadaver neck lengths were measured in this manner; the remaining 18 measurements were not obtained due to previous dissection of the region.

\section{Statistical analysis}

Data were recorded in Microsoft Excel and exported into SPSS. Per cent occurrence and average branch length per category were initially calculated. The two sample Student's $t$-test was used to compare the average length of the AELB on the left and right side, as well as average branch length between males and females, and the average neck lengths of those with and without ELB. The $\chi^{2}$ test was utilised, assuming equal incidence, to assess the occurrence of the ELB on the left and right. Least squares linear regression on logged values was used to analyse the individual relationships between branch length and neck length. Finally, Spearman's rank correlation compared gender and branch length.

\section{RESULTS}

Extralaryngeal branching was present in $77.78 \%$ (35/45) of specimens, with branching occurring $77.14 \%(27 / 35)$ of the time on the left and $54.29 \%$ (19/35) on the right (Table 1). Overall average length of the AELB was $20.57 \mathrm{~mm}$, in accordance with many previous studies, which averaged $20 \mathrm{~mm}$ from the point of bifurcation to the cricothyroid membrane (range 6-40 mm) [3, 11, 16-19]. Branching occurred in $76 \%(19 / 25)$ of male specimens and in $80 \%(16 / 20)$ of female specimens. Bilateral branching was observed in $31.43 \%(11 / 35)$ of specimens.

Mean length of the AELB on the left was $22.44 \mathrm{~mm}$ (range 11.55-28.06 $\mathrm{mm}$ ), while mean length on the right was $18.71 \mathrm{~mm}$ (range $9.20-35.48 \mathrm{~mm}$ ). This was a statistically significant difference between right and left AELB length $(p<0.05)$.

Although females more frequently exhibited an $E L B$, we did not find a significant difference in ELB incidence between genders. There also was no significant difference between the average branch lengths in males compared to females (males $20.08 \mathrm{~mm}$; females $20.78 \mathrm{~mm}$ ). Within males and females AELB length was comparable on both sides, although average branch length was greater on the left than the right for both genders (female right $19.39 \mathrm{~mm}$, left $22.17 \mathrm{~mm}$; male right $17.97 \mathrm{~mm}$, left $21.74 \mathrm{~mm}$; Table 2).

A significant difference between the neck lengths of individuals with and without ELB was evident $(p<0.05)$. The average neck length with ELB was $110.24 \mathrm{~mm}$, while the average neck length without ELB was $96.68 \mathrm{~mm}$.

When analysed individually, neither gender $\left(r^{2}=0.026\right)$ nor neck length $\left(r^{2}=0.062\right)$ correlated 
Table 1. Rate of occurrence of the anterior extralaryngeal branch of the recurrent laryngeal nerve

\begin{tabular}{ll}
\hline Overall occurrence & $77.78 \%(35 / 45)$ \\
Percent bilaterality & $31.43 \%(11 / 35)$ \\
Percent occurring on right & $54.29 \%(19 / 35)$ \\
Percent occurring on left & $77.14 \%(27 / 35)$ \\
Incidence in males & $76.00 \%(19 / 25)$ \\
Incidence in females & $80.00 \%(16 / 20)$ \\
\hline
\end{tabular}

Table 2. Average anterior extralaryngeal branch (AELB) and neck lengths

\begin{tabular}{ll}
\hline Average AELB length & $20.57 \mathrm{~mm}$ \\
Average right AELB length & $18.71 \mathrm{~mm}$ \\
Average left AELB length & $22.44 \mathrm{~mm}$ \\
Average female AELB length: & $20.78 \mathrm{~mm}$ \\
Right & $19.39 \mathrm{~mm}$ \\
Left & $22.17 \mathrm{~mm}$ \\
Average male AELB length: & $20.08 \mathrm{~mm}$ \\
Right & $17.97 \mathrm{~mm}$ \\
Left & $21.74 \mathrm{~mm}$ \\
Average female neck length & $105.39 \mathrm{~mm}$ \\
Average male neck length & $106.36 \mathrm{~mm}$ \\
Average neck length with AELB & $110.24 \mathrm{~mm}$ \\
Average neck length without AELB & $96.68 \mathrm{~mm}$ \\
\hline
\end{tabular}

with AELB length. Gender and neck length are also unrelated, as males displayed an average neck length of $106.36 \mathrm{~mm}$ and the average neck length in females was $105.39 \mathrm{~mm}$.

\section{DISCUSSION}

The course of the RLN through the neck is of vital importance to head and neck surgeons, particularly during thyroidectomy. The surgeon should take great care to ascertain the presence and location of ELB, in particular the AELB, as it has recently become evident that this branch, when present, carries all of the motor fibres supplying the laryngeal constrictors [10, 18]. This study focused on how one might predict which individuals will have ELB of the RLN.

The incidence of the ELB has proven to conflict among studies, with some reporting an incidence as high as $92.7 \%$ [23] and others as low as $18.5 \%$ [3]. In turn, some have reported a higher incidence on the right $[1,3,9]$, while others report no significant difference between the sides [18]. Bilateral bifurca- tions are less common than unilateral bifurcations. The RLN is asymmetric throughout its course in both the thorax and neck, which suggested that additional asymmetry could be present among its branches. In the present study, overall ELB incidence was $77.78 \%$. ELB occurrence on the right was $54.29 \%$, while left ELB was present in $77.14 \%$ of our sample, which did not result in a statistically significant difference in the incidence per side. Several previous studies have found that there is a significant difference in occurrence between sides $[1,3,9]$, while others have found a relatively equal incidence for each side [18]. Thus, our findings agree with some of the previous studies, but conflict with others, suggesting sample, and possibly population, specific differences in occurrence.

In addition, we were interested in determining if the length of the right and left AELB differed significantly. In our study, the average point of bifurcation of the RLN into AELB and PELB was $18.71 \mathrm{~mm}$ on the right and $22.44 \mathrm{~mm}$ on the left, indicating that the average person will have a longer AELB on the left. Surgeons should begin searching for left ELB earlier along the course of the left RLN. Though a difference of approximately $4 \mathrm{~mm}$ in length may initially seem of little consequence, when one considers the limited visual field available to a surgeon during thyroidectomy, $4 \mathrm{~mm}$ becomes increasingly relevant.

Anterior extralaryngeal branch length and sidedness in the male and female subsamples were also compared. We did not find an appreciable difference between AELB lengths per side, although our values did approach significance. These values indicated that the sample size within our subsample was not large enough, since individual genders did not differ significantly between right and left AELB length, but when the genders were pooled, the difference did attain significance.

The relationship between individual neck length and the incidence and length of the AELB was also a focus. We sought to determine this relationship because a measurement of the neck would be easily accomplished in an office visit, allowing the surgeon to predict the presence of ELB prior to surgery. In our sample, the average neck length of those with ELB was $110.24 \mathrm{~mm}$, while those without ELB had an average neck length of only $96.68 \mathrm{~mm}$. Thus, we concluded that those with longer necks are more likely to have ELB than those with shorter necks. However, we did not find a significant correlation between the neck length and the length of the AELB. 
This suggests that while we may be able to use the neck length of a patient to determine whether ELB is present, we cannot necessarily determine at what point the RLN will bifurcate based on the neck length of the individual.

We were also interested in determining if there was a difference in the ELB occurrence and the AELB length in males versus females. In this sample, we did not find any significant differences between genders. The average length of both the AELB and the neck were remarkably similar in males and females, thus allowing us to conclude that gender has a negligible effect on these two variables. In addition, the incidence of ELB between males and females did not differ to a significant degree, suggesting that the AELB is as likely to occur in males as females, and that, when present, the bifurcation can be located at approximately the same position along the course of the RLN $(\sim 20 \mathrm{~mm}$ from the cricothyroid membrane) in both genders.

\section{Limitations of the study}

One potential limitation of this study was the sample size. The previous studies conducted in surgical samples observed hundreds of individuals $[3,4,9-12$, 18] while ours contained 90 RLNs. However, the ability to dissect more invasively with cadaveric specimens allowed us to follow the course of the RLN completely and obtain greater certainty of ELB incidence. In addition, our sample size was decreased by the exclusion criterion involving thyroid gland pathology, but it was essential to exclude these specimens rather than potentially report AELB lengths greater than those present in individuals without any pathology.

\section{CONCLUSIONS}

Our study attempted to ascertain whether occurrence and branch length of the AELB of the RLN could be predicted by gender, neck length, or sidedness. We found significant differences in the neck lengths of those with and without ELB and have concluded that neck length can be used as a predictor of the presence of ELB. We have also discovered that the $A E L B$, when present, will be longer on the left than the right. Head and neck surgeons should be aware of these trends in their patients and consider taking a neck measurement prior to operation to determine if the patient is likely to display ELB. However, surgeons should be aware that ELB of the RLN can, and does, occur in many different types of patients and be mindful of this when performing surgical procedures.

\section{REFERENCES}

1. Beneragama T, Serpell JW. Extralaryngeal bifurcation of the recurrent laryngeal nerve: a common variation. ANZ J Surg. 2006; 76(10): 928-931, doi:10.1111/j.14452197.2006.03899.x, indexed in Pubmed: 17007624.

2. Cakir BO, Ercan I, Sam B, et al. Reliable surgical landmarks for the identification of the recurrent laryngeal nerve. Otolaryngol Head Neck Surg. 2006; 135(2): 299-302, doi: 10.1016/j.otohns.2006.03.026, indexed in Pubmed: 16890087.

3. Casella C, Pata G, Nascimbeni R, et al. Does extralaryngeal branching have an impact on the rate of postoperative transient or permanent recurrent laryngeal nerve palsy? World J Surg. 2009; 33(2): 261-265, doi: 10.1007/s00268008-9832-1, indexed in Pubmed: 19023612.

4. Cernea CR, Hojaij FC, De Carlucci D, et al. Recurrent laryngeal nerve: a plexus rather than a nerve? Arch. Otolaryngol. Head Neck Surg. 2009; 135(11): 1098-1102, doi: 10.1001/ archoto.2009.151, indexed in Pubmed: 19917921.

5. Chiang FY, Lu IC, Chen HC, et al. Anatomical variations of recurrent laryngeal nerve during thyroid surgery: how to identify and handle the variations with intraoperative neuromonitoring. Kaohsiung J. Med. Sci. 2010; 26(11): 575-583, doi: 10.1016/S1607-551X(10)70089-9, indexed in Pubmed:21126710.

6. Eckel HE, Sittel C, Zorowka P, et al. Dimensions of the laryngeal framework in adults. Surg Radiol Anat. 1994; 16(1): 31-36, doi: 10.1007/bf01627918, indexed in Pubmed: 8047966.

7. Gregg RL. Avoiding injury to the extralaryngeal nerves. Ann. Otol. Rhinol. Laryngol. 1957; 66(3): 656-678, doi: 10.1177/000348945706600305, indexed in Pubmed: 13488341.

8. Kahane JC. Growth of the human prepubertal and pubertal larynx. J Speech Hear Res. 1982; 25(3): 446-455, doi: 10.1044/jshr.2503.446, indexed in Pubmed: 7176619.

9. Kandil E, Abdel Khalek M, Aslam R, et al. Recurrent laryngeal nerve: significance of the anterior extralaryngeal branch. Surgery. 2011; 149(6): 820-824, doi: 10.1016/j. surg.2011.02.012, indexed in Pubmed: 21497872.

10. Kandil E, Abdelghani S, Friedlander P, et al. Motor and sensory branching of the recurrent laryngeal nerve in thyroid surgery. Surgery. 2011; 150(6): 1222-1227, doi: 10.1016/j. surg.2011.09.002, indexed in Pubmed: 22136844.

11. Katz $A D$. Extralaryngeal division of the recurrent laryngeal nerve. Report on 400 patients and the 721 nerves measured. Am. J. Surg. 1986; 152(4): 407-410, doi: 10.1016/00029610(86)90313-2, indexed in Pubmed: 3766872.

12. Katz $A D$, Nemiroff P. Anastamoses and bifurcations of the recurrent laryngeal nerve--report of 1177 nerves visualized. Am Surg. 1993; 59(3): 188-191, indexed in Pubmed: 8476158.

13. Maranillo $E$, Leon $X$, Orus $C$, et al. Variability in nerve patterns of the adductor muscle group supplied by the recurrent laryngeal nerve. Laryngoscope. 2005; 115(2): 358-362, doi: 10.1097/01.mlg.0000154745.78808.02, indexed in Pubmed: 15689766.

14. Maue WM, Dickson DR. Cartilages and ligaments of the adult human larynx. Arch Otolaryngol. 1971; 94(5): 432-439, doi:10.1001/archotol.1971.00770070678008, indexed in Pubmed: 5114952. 
15. Morrison LF. Recurrent laryngeal nerve paralysis; a revised conception based on the dissection of one hundred cadavers. Ann. Otol. Rhinol. Laryngol. 1952; 61(2): 567-592, doi: 10.1 177/000348945206100228, indexed in Pubmed: 14944155.

16. Nemiroff PM, Katz AD. Extralaryngeal divisions of the recurrent laryngeal nerve. Surgical and clinical significance. Am. J. Surg. 1982; 144(4): 466-469, doi: 10.1016/00029610(82)90425-1, indexed in Pubmed: 7125080.

17. Nguyen M, Junien-Lavillauroy C, Faure C. Anatomical intralaryngeal anterior branch study of the recurrent (inferior) laryngeal nerve. Surg Radiol Anat. 1989; 11(2): 123-127, doi: 10.1007/bf02096467, indexed in Pubmed: 2763003.

18. Serpell JW, Yeung MJ, Grodski S. The motor fibers of the recurrent laryngeal nerve are located in the anterior extralaryngeal branch. Ann. Surg. 2009; 249(4): 648-652, doi: 10.1097/SLA.0b013e31819ed9a4, indexed in Pubmed: 19300223.

19. Steinberg JL, Khane GJ, Fernandes CM, et al. Anatomy of the recurrent laryngeal nerve: a redescription.
J Laryngol Otol. 1986; 100(8): 919-927, doi:10.1017/ s0022215100100325, indexed in Pubmed: 3746108.

20. Sunderland S, Swaney WE. The intraneural topography of the recurrent laryngeal nerve in man. Anat. Rec. 1952; 114(3): 411-426, doi:10.1002/ar.1091140303, indexed in Pubmed: 12996881.

21. Wafae N, Vieira MC, Vorobieff A. The recurrent laryngeal nerve in relation to the inferior constrictor muscle of the pharynx. Laryngoscope. 1991; 101(10): 1091-1093, doi: 10.1288/00005537-199110000-00011, indexed in Pubmed: 1921637.

22. Williams AF. The recurrent laryngeal nerve and the thyroid gland. J Laryngol Otol. 1954; 68(11): 719-725, doi: 10.1017/s0022215100050192, indexed in Pubmed: 13212256.

23. Yalçin B, Ozan H. Extralaryngeal bifurcation of the recurrent laryngeal nerve. ANZ J Surg. 2007; 77(4): 306, doi: 10.1111/j.1445-2197.2007.04044.x, indexed in Pubmed: 17388844. 